\title{
Multifunctional, Optical Sensor for Analysis of Dynamic Pupillary Light Reflex
}

\author{
K. RóżAnOWski ${ }^{a, *}$, J. LEWANDOWski $^{a}$ And K. Murawski ${ }^{b}$ \\ ${ }^{a}$ Military Institute of Aviation Medicine, Aviation Bioengineering Department, \\ Z. Krasińskiego 54, 01-755 Warsaw, Poland \\ ${ }^{b}$ Military University of Technology, Institute of Teleinformatics and Automatics, \\ S. Kaliskiego 2, 00-908 Warsaw, Poland \\ (Received May 12, 2014; in final form January 26, 2015)
}

\begin{abstract}
The aim of the publication is to describe the multifunctional optical sensor for analysis of dynamic pupillary light reflex developed by the authors which could be used as an objective indicator of human fatigue. The authors utilized knowledge gained from past experimental studies to develop a sensor, which along with custom software for detection of parameters of dynamic pupillary reflex provides a quick analysis of the human psychophysiological condition. Furthermore, the obtained parameters may also be utilized for medical purposes. The authors present the results of pilot studies prior to the development process of the sensory solution. The obtained result show that the type of light stimulation of the retina has a significant impact on the dynamic parameters of the pupillary reflex. The results played also an important role in shaping the structural assumptions of the sensor, so that it can serve as an indicator of the human fatigue level. The article includes results of experimental research conducted during the testing process of the sensor using various parameters of frequency light excitations, luminance and chrominance.
\end{abstract}

DOI: 10.12693 /APhysPolA.127.723

PACS: 42.66.Ct, 42.30.Va, 07.05.Pj, 07.07.Df

\section{Introduction}

Described sensor is a combination of hardware and software solutions. Installed specially designed goggle housing allows for both stationary and mobile use.

This class of optical sensors, using vision-based image analysis, is used in pupillometry and enables identification of eyeball movement, parameters describing the dynamics of the eyeball covering and dynamic pupil change reaction. The evaluation of pupil motion provides functional information about the autonomous nervous system $[1,2]$. Pupillary light reflex (PLR) is the iris's response to changes in the intensity of light incidence on the retina (Fig. 1). This reflex is modulated by the accommodation state of the eye, and factors of sensory and emotional origins. Effectors are contractor and dilator muscles of the iris that try to maintain a constant level of retina illumination by changing the size of the pupil. Time characteristic of the pupillary reflex depends on the state of the two antagonistically acting systems of the autonomous nervous system (ANS): sympathetic nervous system (SNS) and parasympathetic nervous system (PNS).

Multi-functional optical sensor allows to programmatically control all the lighting parameters of the pupil that may have an effect on its behavior. The wavelength of the light source illuminating the pupil and its intensity are both subject to control. Also the background light in case

*corresponding author; e-mail: krozan@wiml.waw.pl

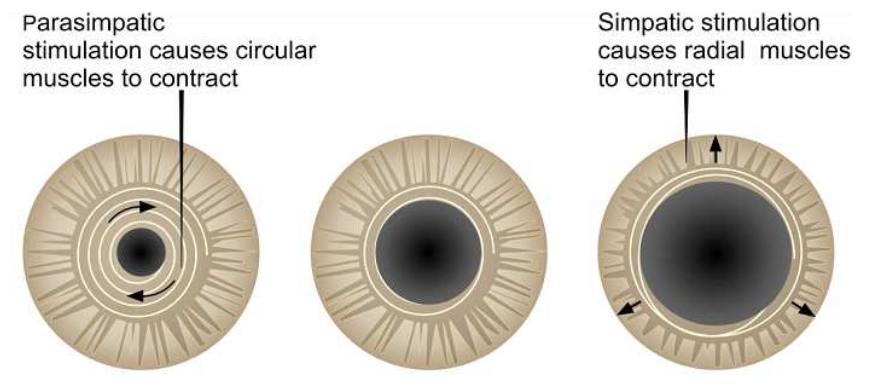

Fig. 1. The iris reaction mechanism.

of research conducted in a darkroom is variable. You can program any combination of luminance and chrominance parameters of the background light and the light illuminating the pupil. The light excitation sequence in terms of their number and duration is fully adjustable. Both of these parameters can be tuned with virtually no restrictions. Thanks to duplicated measuring circuits and the use of two independent sensors, also concurrent analysis of pupillary behavior for different types of stimulation as well as consensus behavior analysis are possible.

\section{Dynamic pupillary reflex process}

Pupillometry was initially used in ophthalmology [3]. The size of the pupil is crucial for the interpretation of perimetry of the visual field, electroretinography and the preoperative assessment for refractive surgery [4]. It is also utilized in neuro-ophthalmology for the assessment of lesions of the afferent or efferent nervous pathways [5]. The PLR has already been studied to detect a PNS in different conditions: alcoholism, diabetes, AIDS, 
depression, anxiety, drug addiction and schizophrenia. Pupillography is also being utilized in research on the Alzheimer and Parkinson disease [6]. Since pupillary dilation is regulated autonomically, an effort was made to use this method as a valid measure of pain [7]. Research using pupillometry is being carried out to assess alertness in hypersomnolent patients. Observation of pupil behavior is also used in clinically sleepy patients [8], as well as in the assessment of cardiac autonomic function in sport athletes [9]. As shown, this method has a variety of clinical applications. Furthermore, the identification of pupil dynamics is also the subject of research concerning the monitoring of various areas of human behavior [10-12].

Current literature lacks publications containing test results showing the dependence of the PLR parameters on the variable level of sensory and mental fatigue. There are several predefined parameters describing the PLR. Parameters that were used in this analysis are those provided by $\mathrm{F}^{2} \mathrm{D}$ Fit-For-Duty device by AMTech, which was used in this research. A detailed description of the determined parameters exists in the earlier work of the authors [13-15].

Pupillary reflex dynamics phenomenon can be described by rheological models which describe behavior of bodies under strain for the description of which perfectly elastic body models cannot be used. An important phenomenon in this case is creeping meaning the growth of deformation induced constant, normal strain to which the body is subjected. They can be described by the Kelvin-Voight model

$$
\varepsilon(t)=\frac{\sigma_{0}}{E}\left(1-e^{\frac{-E}{\eta} t}\right),
$$

where $\varepsilon(t)$ - total strain, $\sigma_{0}$ - initial strain, $E-$ Emodulus, $\eta$ - dynamic viscosity, $t$ - time.

The two-piece model is too simple to describe the actual biological materials. Therefore subsequent elements are added until satisfactory mapping accuracy is achieved. A generalized form of the model is represented by the equation

$$
\varepsilon(t)=\sigma_{0}\left(\frac{1}{E_{0}}+\sum_{i=1}^{n} \frac{1}{E_{i}}\left(1-e^{\frac{-i}{\tau_{i}}}\right)+\frac{1}{\eta_{0}}\right),
$$

where $\tau_{i}$ - relaxation time.

\subsection{Design of the model describing the PLR phenomenon}

Since the two iris muscles play essential roles in controlling the pupil size, their dynamic properties must be considered in the PLR model (Fig. 2). The constrictor and dilator muscles could be modeled as viscoelastic materials using Kelvin-Voigt model in a circular and linear shape, respectively. The movement of the iris muscles is governed by three forces: passive muscle elastic force, viscous resistance, and the active forces generated by ANS modulation. The constrictor is modulated by the parasympathetic system, whereas the dilator is modulated by the sympathetic system. Let the radius of constrictor be $r_{c}$, and the length of dilator muscle be $r_{d}$. Assuming the constrictor has a circular shape with a perimeter of $2 \pi r_{c}$, the dynamic equations can be constructed for constrictor and dilator as

$$
\begin{aligned}
& m_{c} \frac{\mathrm{d}^{2} r_{c}}{\mathrm{~d} t^{2}}=-2 \pi P_{e c}+P_{v c}-\frac{F_{p}(t)}{2 \pi}, \\
& m_{d} \frac{\mathrm{d}^{2} r_{d}}{\mathrm{~d} t^{2}}=-P_{e d}+P_{v d}-F_{s}(t),
\end{aligned}
$$

where $m_{c}$ - the mass for constrictor (in $\mathrm{mg}$ ), $m_{d}$ - the mass for dilator (in $\mathrm{mg}$ ), $P_{e c}$ - the viscous resistance in the constrictor, $P_{e d}-$ the viscous resistance in the dilator.

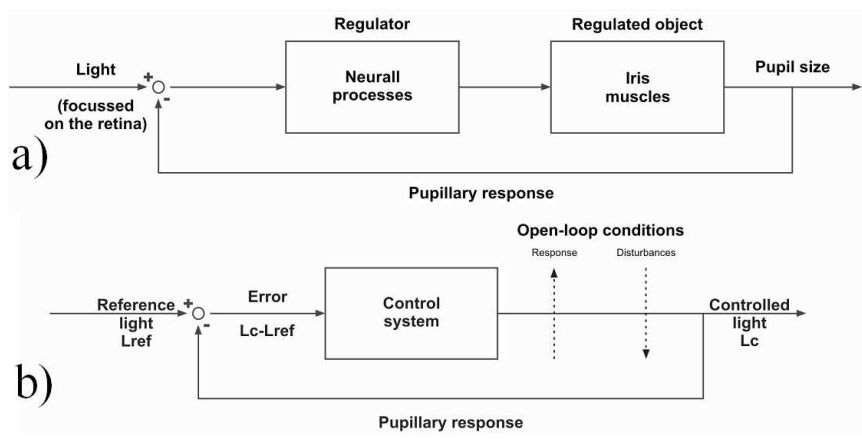

Fig. 2. Dynamic model of PLR with negative feedback.

The passive iris muscle elasticity was modeled as a second-order equation of muscle length based on experimental observation [16]:

$$
\begin{aligned}
& P_{e c}=\left\{\begin{array}{cl}
k_{c}\left(r_{c}-l_{0 c}\right)^{2}, & r_{c} \geq l_{0 c}, \\
0, & r_{c}<l_{0 c},
\end{array}\right. \\
& P_{e d}=\left\{\begin{array}{cl}
k_{d}\left(r_{d}-l_{0 d}\right)^{2}, & r_{d} \geq l_{0 d}, \\
0, & r_{d}<l_{0 d},
\end{array}\right.
\end{aligned}
$$

where $k_{c}, k_{d}$ - elastic constants, $l_{0 c}$ - the radius of the constrictor at the rest, $l_{0 d}$ - the dilator muscle resting length.

$P_{v c}$ and $P_{v d}$ were modeled as a linear function of velocity [17]:

$$
\begin{aligned}
& P_{v c}=-D_{c} \frac{\mathrm{d} r_{c}}{\mathrm{~d} t}, \\
& P_{v d}=-D_{d} \frac{\mathrm{d} r_{d}}{\mathrm{~d} t},
\end{aligned}
$$

where $D_{c}, D_{d}$ - viscous constants.

The $F_{p}(t)$ and $F_{s}(t)$ in (2.3) are the muscle forces originated from the parasympathetic and sympathetic system induced by light stimulus, respectively. These are effective forces that could be affected by both the afferent and efferent pupillary pathway. For short-pulse stimulus, the forces originated from the parasympathetic and sympathetic system were assumed to be square-wave pulses with different delays, $\tau_{p}$ and $\tau_{s}$ and durations, $\Delta t_{p}$ and $\Delta t_{s}[18]:$ 


$$
\begin{aligned}
& F_{p}(t)=\left\{\begin{array}{cc}
f_{p}+f_{p 0}, & \left(\tau_{p} \leq t \leq \tau_{p}+\Delta t_{p}\right) \\
f_{p 0}, & \left(t<\tau_{p}, t>\tau_{p}+\Delta t_{p}\right)
\end{array}\right. \\
& F_{s}(t)=\left\{\begin{array}{cc}
f_{s}+f_{s 0}, & \left(\tau_{s} \leq t \leq \tau_{s}+\Delta t_{s}\right) \\
f_{s 0}, & \left(t<\tau_{s}, t>\tau_{s}+\Delta t_{s}\right)
\end{array}\right.
\end{aligned}
$$

where $f_{p 0}, f_{s 0}$ - the muscle forces originated from the parasympathetic and sympathetic systems at the resting condition.

\section{Experimental phase preceding the construction of a PLR sensor}

The authors have conducted experimental studies of a pupillary reflex sensor model [13]. Preliminary results confirmed the research hypothesis that it is possible to assess the subjective sense of fatigue level with the parameters describing the dynamic pupillary reflex. Tests were conducted using FlexComp diagnostic equipment which offers 10 high-speed channels (2048 samples/s) and communicates with Thought Technology sensors. Due to the ability to view data in an online mode, subjects were monitored for their ability to participate in subsequent phases of the experiment. Their psychophysical ability was verified using APK apparatus (visual-motor coordination test device). An algorithm for the measurement and evaluation of physiological parameters was used in the study $[19,20]$. The experiment was conducted in accordance with the developed methodology after obtaining the consent of the Commission of Bioethics [21].

The objective was to determine the relationship between PLR parameters and the results of the pupillographic sleepiness test (PST). Due to the fact that the PST is a well-established method utilized in sleep research/medicine and consists of an 11-minute recording of pupil diameter by infrared video pupillography, the pupillary unrest index (PUI) factor was used as a reference for verification of analyzed pupillary reflex parameters in the assessment of the level of sleepiness and reduced concentration.

The experimental results were used to clarify the requirements for the prototype PLR sensor described in the article.

\section{Multi-functional optical sensor to monitor pupillary light reflex}

The developed multifunctional optical sensor prototype allows software control of all the parameters of the pupil illumination that may affect its behavior. The wavelength of the light source illuminating the pupil and its intensity are both subject to control. Also the background light in case of research conducted in a darkroom is variable. You can program any combination of luminance and chrominance parameters of the background light and the light illuminating the pupil. The light excitation sequence in terms of their number and duration is fully adjustable. Both of these parameters can be tuned with virtually no restrictions. Thanks to duplicated measuring circuits and the use of two independent sensors, also concurrent analysis of pupillary behavior for different types of stimulation as well as consensus behavior analysis are possible.

The designed solution allows static as well as dynamic pupillometric measurements. In terms of static pupillometry it is possible to set photopic, mesopic, and scotopic lighting.

Under photopic vision conditions only cones (receptors enabling color vision) are involved while rods (receptors that recognize differences in light intensity only in almost complete darkness, monochromatic), are not. As the intensity of the ambient light decreases, photopic vision goes into a transition phase called mesopic vision (partial loss of color perception), to finally reach the minimum operating status of the human eye called scotopic vision (world in a grayscale). Three types of cones provide the day vision in the human eye, showing the greatest sensitivity at wavelengths corresponding to 420,534 , and $564 \mathrm{~nm}$. Spectral maximum for night vision is located at $498 \mathrm{~nm}$ (Fig. 3). The cones have a significantly lower sensitivity (of the order of $600 \mathrm{~lm} / \mathrm{W}$ ) than the rods (about $1700 \mathrm{~lm} / \mathrm{W}$ ).

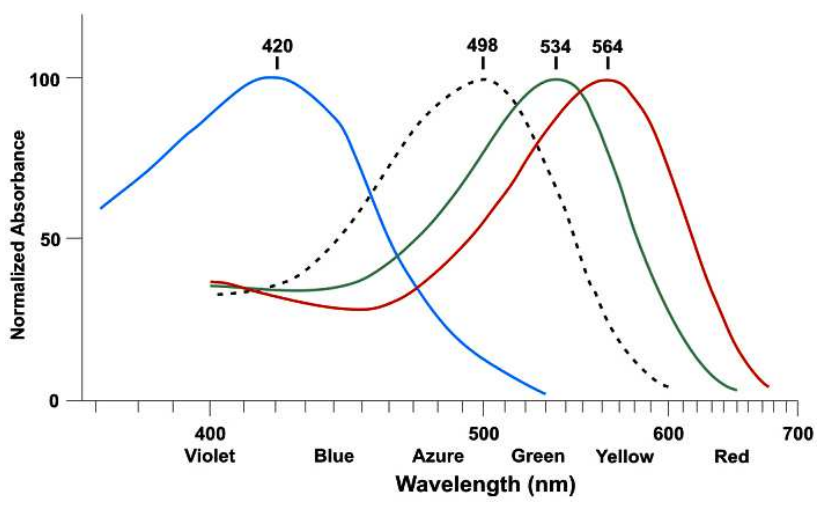

Fig. 3. Spectral maxima for various light wavelengths.

In the dynamic pupillometry measurement mode lighting conditions change smoothly and the image is recorded simultaneously for both eyes. Also the reaction of the pupil to the flashes is examined. The test result contains time analysis of pupil response to changes in illumination, the maximum and minimum diameter of the pupil and the pupil response time. Advanced parameters of the examination are also available, among others: latency, amplitude of shrunken pupil shrink rate, etc.

Optical sensor for dynamic pupillary light reflex was designed in such a way that it could be implemented in goggles' housing. Two adjustable side cameras that track the eyeballs are mounted in the measuring heads together with infrared illuminators and filters. Side cameras observe the eye image reflected in the infrared mirror. The IR mirror is rigidly installed in the housing. The front part of the goggle can be covered by a lid which prevents light from entering the interior of the goggles. 
The lid may be attached to the goggle with neodymium magnets embedded in the covers which attract the metal inserts embedded in the cover.

The two MU9PM side cameras work with a resolution of $2592 \times 1944$ pix ( $5 \mathrm{MP}$ ) with a dynamic of $70 \mathrm{~dB}$ and $38 \mathrm{~dB}$ SNR. Frame-rate can reach up to $232 F_{p}$ 's, depending on the resolution settings. Cameras are aimed at the pupils and transmit the image directly into a computer system using a USB connection. Side cameras coupled with configurable illuminators are equipped with a set of three-color LEDs (Fig. 4).

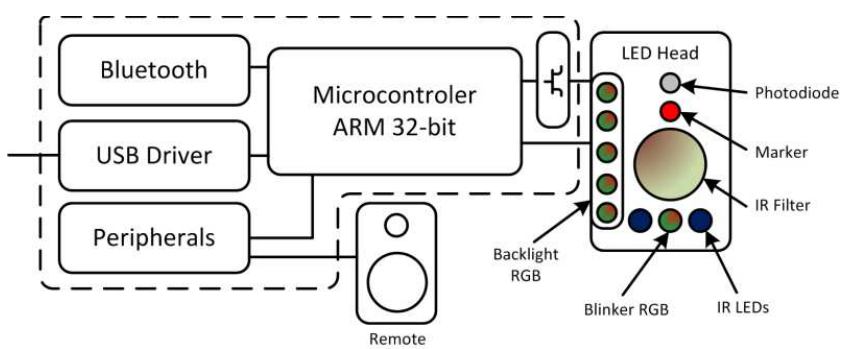

Fig. 4. Functional block diagram of the control part of the sensor.

Illuminators are governed by a digital controller. Side cameras observe the pupil's infrared image reflected in IR mirrors. The image visible for the cameras is cut-off because they are separated by an IR filter transmitting only infrared light (BP850-25.5). The pupil is illuminated by IR LEDs placed in the illuminator module. Illuminator is also equipped with five RGB-LED KPFT1616 LEDs arranged in a vertical line on the edge of illuminators to establish the background lighting conditions, one LED located above the camera to induce the pupillary reflex, one red RGB-LED diode which acts as a marker for focusing your vision on, and a photodiode for measuring the intensity of light emitted by the head, Fig. 5. All LEDs and IR's brightness is adjustable with the help of a digital controller. The sensor's controller is made using a STM32F103VET6 32-bit ARM Cortex-M3 microcontroller. The controller connects to your computer via USB and allows individual control of each LED installed in the goggles. In addition to the control via computer, the driver has mechanisms regulating the LED brightness and frequency of flicker implemented. In addition it allows for automatic tuning of diodes via computer or an included remote control. To ensure simultaneous adjustment of brightness and flicker of the LEDs in the optical heads each of them have dedicated MOS transistor. Adjusting the flicker of the LEDs is exercised simultaneously for all three channels of the particular RGB LED. LED IR-LED HIRB543G-D diodes were used to illuminate the image in infrared. The whole unit is powered from a standard USB port of your computer. The brightness of the LEDs is controlled by PWM. The controller uses 20 PWM hardware channels to govern the LEDs.

The LEDs are placed in the optical heads so as to allow the determination of the sight focal point (the so-called LED marker), blinding the observer (LED blinker), ade- quate lighting of the image tracked by the IR camera and visible light background lighting (so called LED backlight). The diodes in the optical head are placed on plates with appropriate holes through which the IR camera observes image of the pupil in infrared.
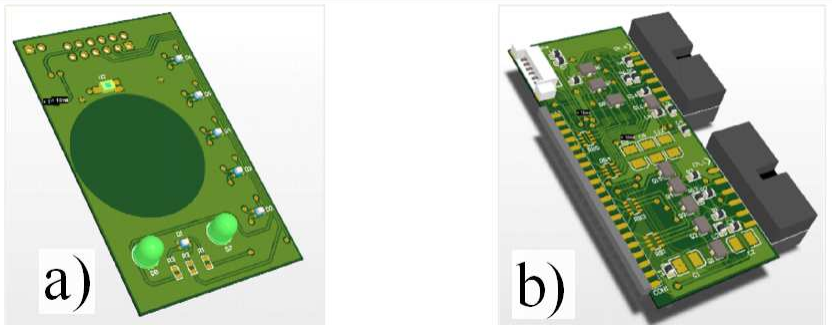

Fig. 5. 3D model of the PCB: (a) with the illuminator LEDs, (b) controller.

An executive plate with MOS drivers was distinguished in the part of the controller that handles the LEDs. This plate is attached to the mother board together with a programmed microcontroller and communication interfaces. This solution allows for easy modification of the executive part of the device without the need to redesign the control section equipped with communication interfaces.

The optical head has been tested to determine the power of the emitted light. The maximum measured values for a single eye are: IR - $0.4 \mathrm{~lx}$, marker - $0.012 \mathrm{~lx}$; background color: red $-7.8 \mathrm{~lx}$, green $-3.4 \mathrm{~lx}$, blue $2.6 \mathrm{~lx}$, white $-13 \mathrm{~lx}$. The measurement was performed with a Sonopan FR-100 photometer with a G.L-100 head.

To control the sensor, an application was developed which acts as an image processor based on the open-CV library. When you run the application, a calibration process follows so that the eye and the pupil were in the center. Calibration takes place both via the application (setting the detection threshold of the pupil) and mechanically by means of camera control.

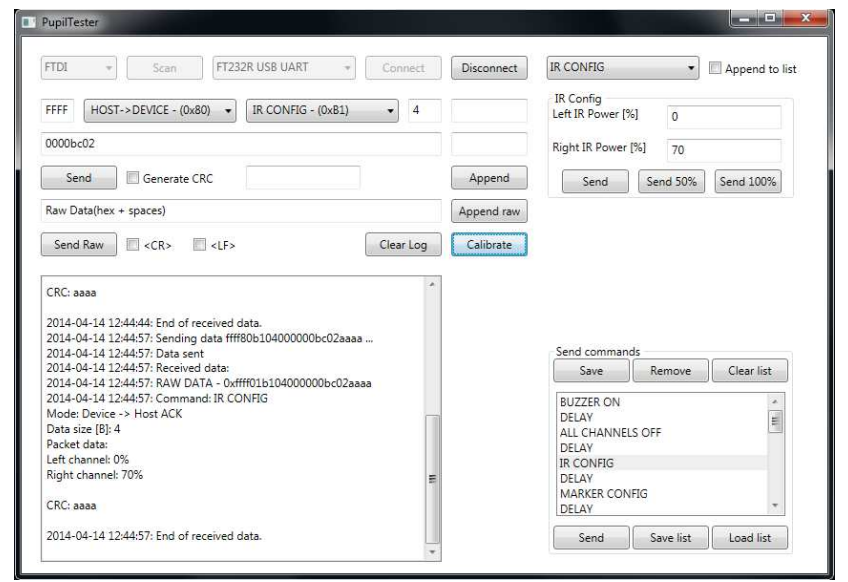

Fig. 6. View of the application window - the sensor controller module.

Once calibrated, you can start conducting any PLR research. A script language was embedded into the application that allows the creation of test scenarios. It is 
possible to create almost any study scenario. Currently, the application has a number of functions in debug mode allowing advanced device control (Fig. 6).

Each action of the application results in saving the data sent to the log file. Configuration of research script is done by creating a list by appending following commands selected from the menu. When the script is ready you can save it in a text file, or load a file generated by you to the application. The scenario describes all the commands and functions the sensor is to exercise i.e. turn on the IR LEDs, turn on the respective RGB-LED diodes with the appropriate brightness, color, flicker frequency and at a given time, or start recording the image.

For data analysis of pupillary reflex a separate application was developed (Fig. 7). After a csv, xls or text data file is loaded, it draws the signal on a chart, a derivative thereof, and calculates the appropriate parameters [13]. Thanks to markers applied to the signal, the researcher can consciously obtain information about which signal points are analyzed and if necessary, correct it by moving the markers on the signal.
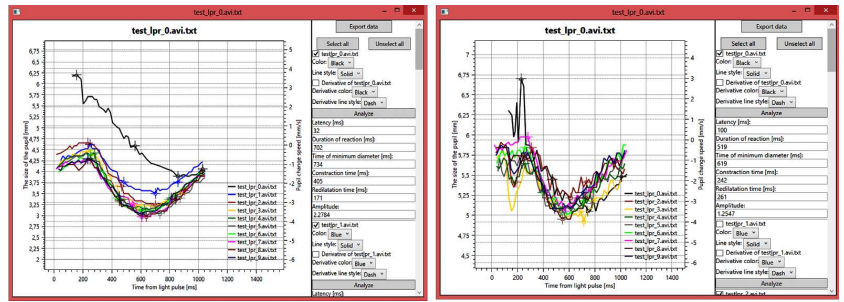

Fig. 7. View of the application window designating sample registration for a series of 10 flashes at the interval of $1 \mathrm{~s}$ with a length of light excitation $50 \mathrm{~ms}$;

(a) white color, (b) red color $\lambda=700 \mathrm{~nm}$.

The analyzer in addition to the signal analysis also computes its derivative and gives its extremes. The data calculated by the analyzer can be exported to a spreadsheet. The software has been developed in the NET 4.0 Windows environment. Each signal recorded by the sensor is saved in image form, as well as signals representing changes in the pupil diameter over time.

The PLR measurement is performed by pressing the "Send" button. The software automatically generates a light pulse of any specified time period, number of sequences and intervals between light's beats. Also, a video showing behavior of the pupil during the measurement is recorded for documentation purposes. The software allows for working "with" and "without" calibrating the camera's optical system. Without calibration, it is possible to determine absolute or relative changes in pupil size. Absolute pupil sizes are measured in pixels (pix.). Relative values do not have a unit of measure, and they are in the $[0,1]$ range. In this case, the starting point for calculations is the highest measured pupil size. In such a configuration, "1" means the largest and " 0 " means the smallest pupil size. Calibration allows for measuring the pupil size in millimeters [13].
Another important aspect was the safety of the sensor. Therefore, the IR illuminator of the sensor was tested and the results are shown in [22].

Software was developed for the constructed design to automatically search the pupil in the image. The pupil searching algorithm uses techniques described in [23-25]. After finding the pupil, its size (width and height) is measured. Finally, because of the lower sensitivity to interference due to eyelid movements, the value equal to the width of the pupil is presented as a result of the measurement.

\section{Test results}

The construction of the multi-functional, optical sensor for analysis of dynamic pupillary light reflex described in point 4 has been subjected to a series of defined tests. The sensor behavior under varying duration of the light pulses 50,150, $150 \mathrm{~ms}$ and varying time intervals $1,5,10 \mathrm{~s}$ was verified. All tests were carried out for the ten beat sequences. The measurement conditions were repetitive, while keeping the 2 min time of eye's accommodation to darkness. All tests were carried out in a quiet room and the tested subject was isolated from all external stimuli.

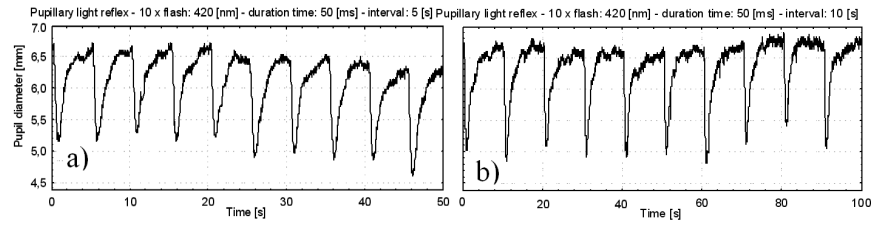

Fig. 8. Sequence of ten pupillary reflexes when excited with light at a wavelength of $420 \mathrm{~nm}$, a pulse duration of $50 \mathrm{~ms}$, and time interval between beats: (a) $5 \mathrm{~s}$, (b) $10 \mathrm{~s}$.

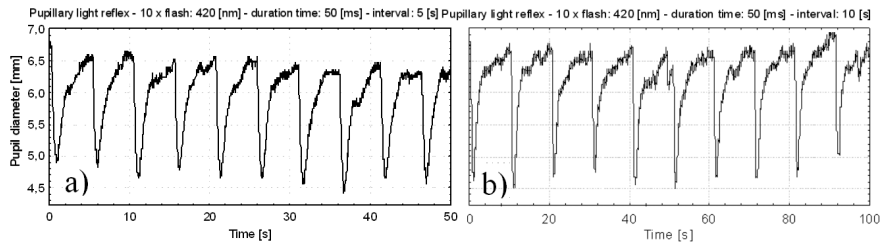

Fig. 9. As Fig. 8 for pulse duration of $150 \mathrm{~ms}$.

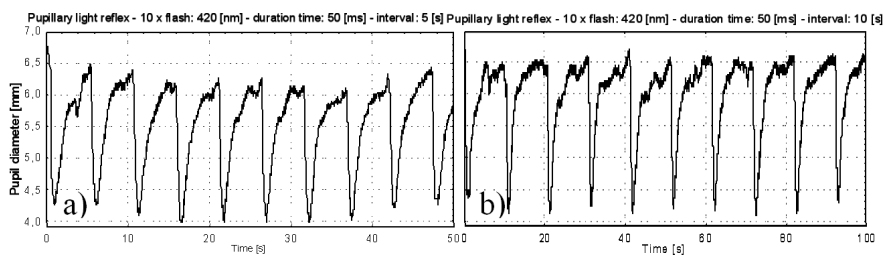

Fig. 10. As Fig. 8 for pulse duration of $250 \mathrm{~ms}$.

The results confirmed the validity of our assumptions and design solutions. The sensor meets the functional and non-functional requirements. The results are reproducible, and the algorithm for pupil detection and determination of the PUI index is working properly. The results shown in Figs. 8-10 are consistent with the prediction of the authors. $1 \mathrm{~s}$ time of intervals between the 
beats is too short to achieve full relaxation of the pupils. However dynamic behavior of the pupils, very similar in successive beats are apparent. The similarity of pupil behavior is more pronounced at longer beats 150 and $250 \mathrm{~ms}$ with time interval of the sequence 5 and $10 \mathrm{~s}$ (Figs. 9, 10).

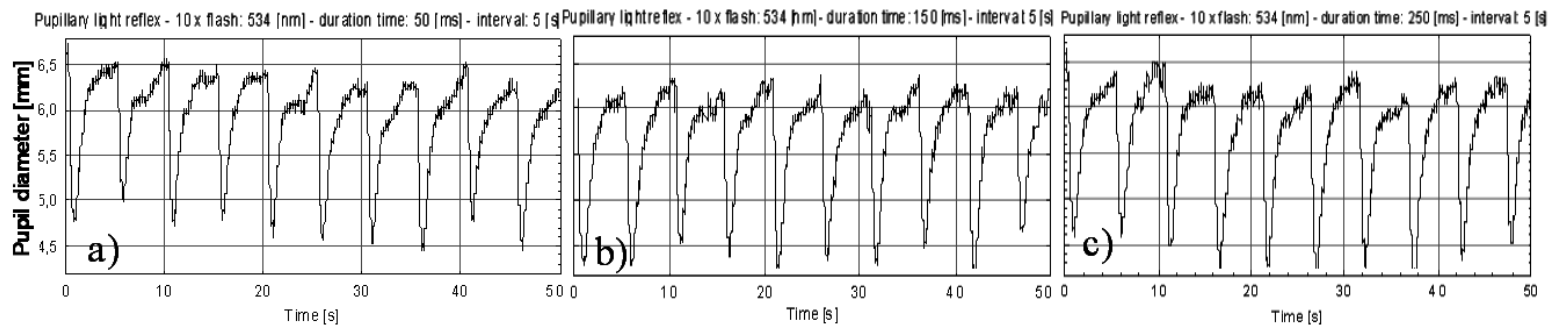

Fig. 11. As Fig. 8 for wavelength of $534 \mathrm{~nm}$, time interval between beats $5 \mathrm{~s}$ and pulse duration of: a) $50 \mathrm{~ms}$, b) $150 \mathrm{~ms}, \mathrm{c}) 250 \mathrm{~ms}$.

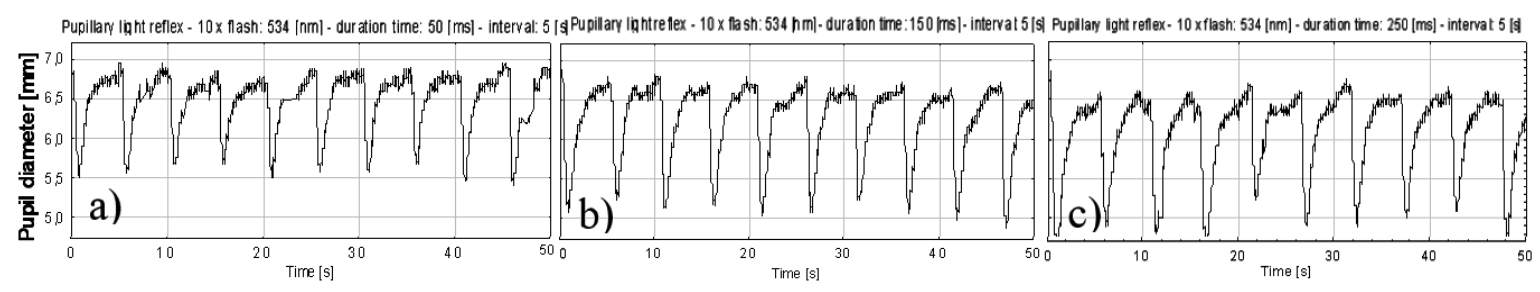

Fig. 12. As Fig. 11 for wavelength of $564 \mathrm{~nm}$.

The achieved reflex repetition is a key effect constituting the quality of the presented solution. Time of the test, in which the examined person was subjected to the influence of light pulses in the range of 10 to $100 \mathrm{~s}$. No changes in ANS activation resulting from subject's fatigue should not occur in such a short period of time. The minimum value of the pupil recorded at the time of its shrinking is smallest under condition of longest light excitation $(250 \mathrm{~ms})$. The research also shows that the minimum time between two successive excitations should preferably be not less than $3 \mathrm{~s}$. The level of activation mechanism of the dynamic pupillary reflex is also influenced by the wavelength of the light falling on the retina. The largest activation of the PLR mechanism occurs with the length of $\lambda=534 \mathrm{~nm}$ (green color), Figs. 11, 12, and Table.

Data analysis of Fig. 13 shows clearly that the pupil size as a consequence of light excitation is by far the lowest in case of the longest excitation $(250 \mathrm{~ms}$ ).

Test examinations with different excitation characteristics were carried out under identical conditions. These preliminary studies on individual persons show that the excitation time has a major impact on the dynamics of pupillary reflex. Analysis of Figs. 11, 12, and Table proves that the highest rate of pupil change is achieved through light excitation at wavelength of $420 \mathrm{~nm}$ and a pulse time of $250 \mathrm{~ms}$. The smallest excitation is induced by light pulses of wavelength $564 \mathrm{~nm}$, not depending on their duration.

The aim of the authors is to find the measurement lighting conditions that will generate the greatest changes

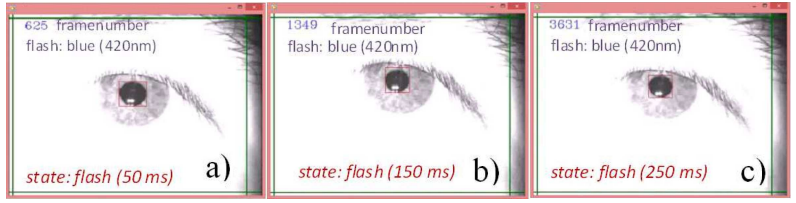

Fig. 13. View of the pupil detection algorithm window for state after excitation (maximum shrinkage) and pulse duration of: a) $50 \mathrm{~ms}$, b) $150 \mathrm{~ms}$, c) $250 \mathrm{~ms}$.

TABLE

Table of minimal average values of pupil diameter after light excitation for a sequence of ten pulses with time interval $5 \mathrm{~s}$

\begin{tabular}{c|c|c|c|c|c|c}
\hline \hline & \multicolumn{2}{|c|}{$\lambda=420[\mathrm{~nm}]$} & \multicolumn{2}{c|}{$\lambda=534[\mathrm{~nm}]$} & \multicolumn{2}{c}{$\lambda=564[\mathrm{~nm}]$} \\
\hline \multirow{2}{*}{$\mathrm{t}_{\text {imp }}[\mathrm{ms}]$} & $\begin{array}{c}\text { Mean } \\
\text { value }\end{array}$ & $\begin{array}{c}\text { Std. } \\
\text { Dev. }\end{array}$ & $\begin{array}{c}\text { Mean } \\
\text { value }\end{array}$ & $\begin{array}{c}\text { Std. } \\
\text { Dev. }\end{array}$ & $\begin{array}{c}\text { Mean } \\
\text { value }\end{array}$ & $\begin{array}{c}\text { Std. } \\
\text { Dev. }\end{array}$ \\
\hline 50 & 5.00 & 0.218 & 4.65 & 0.168 & 5.56 & 0.096 \\
\hline 150 & 4.66 & 0.138 & 4.36 & 0.145 & 5.08 & 0.099 \\
\hline 250 & 4.09 & 0.140 & 4.34 & 0.182 & 4.88 & 0.193
\end{tabular}

in the behavior of PLR in the absence of the need for research under the conditions of complete darkness. Major changes in the pupillary reflex increase the probability of observing discrete behavioral changes of ANS. The results presented in this paper show that the developed optical sensor is very well suited for this purpose.

\section{Conclusions}

Investigation performed by the authors indicates that the results of dynamic pupillary reflex analysis can help identify various states of reduced alertness, decreased 


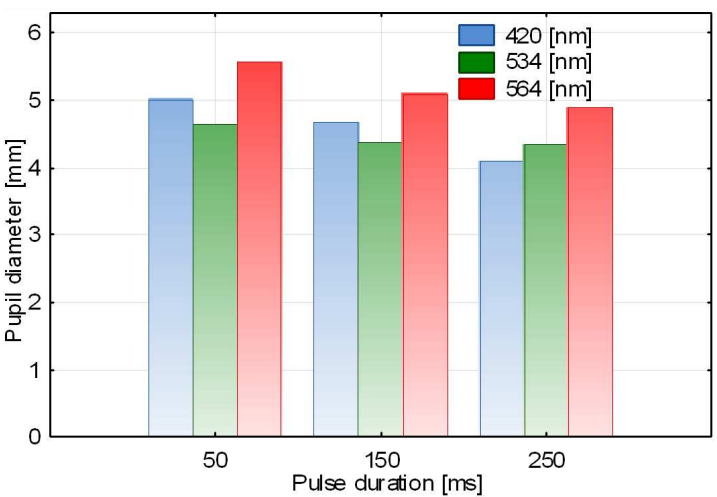

Fig. 14. Characteristic of changes in PLR dynamics depending on the duration of the light stimulus.

concentration, fatigue and drowsiness. High-resolution PLR techniques may become an alternative to the PST, which is the current standard for analysis of the level of drowsiness.

Observation of dynamic pupillary reflex, however, requires the use of high-precision sensors to determine pupil size and high temporal resolution. The authors have developed a solution that offers basically unlimited possibilities of programming sequences of light stimuli in terms of parameters of the light source, background, pulse durations, which may be different for each stimulation (different for each eye), time intervals between beats, the number of beats, parallelism of excitations (excitation of both pupils), consensual reflex analysis (Fig. 14).

This solution has great research potential regarding the search for a fast and effective method to assess the level of fatigue, even under variable ambient lighting.

In order to extend the diagnostic capability of the sensor it is necessary to extend the system with analytical module based on the dynamic model described in Sect. 2 of the paper.

The technique presented in this article is part of a mobile pupillographic device with integrated critical frequency detector, as well as PUI sensor. It is a unique solution that shortens the duration of an objective analysis of fatigue more than 5-fold, in comparison to the PST.

\section{Acknowledgments}

The project was co-financed by the European Union through the European Regional Development Fund under the Innovative Economy Program, Development Project contract no. POIG.01.03.01-10-085/09, and funding by the National Research and Development Centre (NCBR) project DOBR/0038/R/ID2/2013/03.

\section{References}

[1] L.L. Lobato-Rincón, M.C. Cabanillas Campos, J.J. Navarro-Vallas, C. Bonnin-Arias, E. Chamorro, C. Sanchez-Ramos Roda, Adicciones 25, 137 (2013).

[2] G.L. Ferrari, J.L.B. Marques, R. Gandhi, S.R. Heller, F.K. Schneider, S. Tesfaye, H.R. Gamba, BioMed. Eng. OnLine 9, 26 (2010).
[3] A. Kawasaki, R.H. Kardon, Ophthalmol. Clin. North Am. 14, 149 (2001).

[4] R.J. Duffey, D. Leaming, J. Refract. Surg. 21, 87 (2005).

[5] I. Loewenfeld, O. Lowenstein, The Pupil: Anatomy, Physiology, and Clinical Applications, Iowa State University Press, 1993.

[6] D.F. Fotiou, V. Stergioua, D. Tsiptsiosa, C. Litharib, M. Nakouc, A. Karlovasitoua, Int. J. Psychophysiol. 73, 143 (2009).

[7] M.Höfle, R. KenntnerMabiala, P. Pauli, G.W. Alpers, Int. J. Psychophysiol. 70, 171 (2008).

[8] J.W. McLaren, P.J. Hauri, Siong-Chi Lin, C.D. Harris, Sleep Med. 3, 347 (2002).

[9] A. Kaltsatou, E. Kouidi, C. Lithari, N. Koutlianos, F. Fotiou, A. Deligiannis, Int. J. Psychophysiol. 77, 283 (2010).

[10] J.F. Payen, S. Isnardon, J. Lavolaine, P. Bouzat, M. Vinclair, G. Francony, Ann. Fr. AnesthReanim. 31, e155 (2012).

[11] B.J. Wilhelm, A. Widmann, W. Durst, C. Heine, G. Otto, Int. J. Psychophysiol. 72, 307 (2009).

[12] M. Warga, H. Lüdtke, H. Wilhelm, B. Wilhelm, Vis. Res. 49, 295 (2009).

[13] K. Różanowski, K. Murawski, Acta Phys. Pol. A 124, 558 (2013).

[14] J.K. de Souza, M.A. Pinto, P.G. Vieira, J. Baron, C.J. Tierra-Criollo, Comput. Meth. Progr. Biomed. 112, 607 (2013).

[15] M.C. Canver, A.C. Canver, K.E. Revere, D. Amado, J. Bennet, D.S. Chung, Comput. Meth. Progr. Biomed. 113, 221 (2014)

[16] K. Yamaji, T. Yoshitomi, S. Usui, Y. Ohnishi, Vis. Res. 43, 479 (2003)

[17] S. Usui, Y. Hirata, Ann. Biomed. Eng. 23, 375 (1995).

[18] Xiaofei Fan, Gang Yao, IEEE Trans. Biomed. Eng. 58, 37 (2011).

[19] Z. Piotrowski, K. Różanowski, Acta Phys. Pol. A 118, 131 (2010).

[20] M. Czarkowski, M. Mikulska, M. Zebrowski, A. Baran, K. Rozanowski, J. Human Hypertens. 18 , 293 (2004).

[21] M. Czarkowski, K. Różanowski, Sci. Eng. Ethics 15, 201 (2009).

[22] M. Życzkowski, K. Różanowski, K. Murawski, M. Karol, P. Markowski, Acta Phys. Pol. A 122, 942 (2012).

[23] K. Różanowski, K. Murawski, Acta Phys. Pol. A 122 , 874 (2012).

[24] K. Murawski, K. Różanowski, M. Krej, Acta Phys. Pol. A 124, 513 (2013).

[25] K. Murawski, K. Różanowski, Acta Phys. Pol. A 124, 509 (2013). 